\title{
Heavy Probes in Strongly Coupled Plasmas With Chemical Potential
}

\author{
Carlo Ewerz and Andreas Samberg
}

\begin{abstract}
We study the properties of heavy probes moving in strongly coupled plasmas at finite chemical potential. Using the gauge/gravity duality we consider large classes of gravity models consisting in deformed $\mathrm{AdS}_{5}$ spacetimes endowed with Reissner-Nordström-type black holes. We report on our analysis of the screening distance of a quark-antiquark pair, its free energy, and the running coupling. These observables show a certain insensitivity as to which model and deformation is used, pointing to strong-coupling universal behavior. Thus, the results may be relevant for modeling heavy quarkonia traversing a quark-gluon plasma at finite net baryon density, and their suppression by melting.
\end{abstract}

\section{Introduction}

Over the past years, gauge/gravity duality ([1, 2, 3]; see e.g. [4] for a review) has been successfully applied to the physics of the QCD medium created in heavy ion collisions at RHIC and LHC. One of the most prominent theoretical results is the computation of a universal value for the ratio of the shear viscosity $\eta$ to the entropy density $s$ in a large class of strongly coupled deconfined plasmas [5, 6] and the conjecture that this value $(\eta / s=1 /(4 \pi))$ may be a lower bound for all physical substances [7]. On the experimental side, it was found that the Quark-Gluon Plasma (QGP) created at RHIC and LHC has a value of $\eta / s$ in the ballpark of the holographic result, indicating strong coupling at the accessible temperatures.

Carlo Ewerz, e-mail: c . ewerz@thphys . uni-heidelberg.de

Andreas Samberg, e-mail:a.samberg@thphys.uni-heidelberg.de

Institut für Theoretische Physik, Universität Heidelberg, Philosophenweg 16, D-69120 Heidelberg, Germany

ExtreMe Matter Institute EMMI, GSI Helmholtzzentrum für Schwerionenforschung,

Planckstraße 1, D-64291 Darmstadt, Germany 
Beyond current high-energy heavy ion programs, which mainly study the hightemperature, low-chemical potential regime of QCD, a major open question is the phase structure of QCD at nonzero baryon chemical potential. Experimentally, this will be addressed for example at future FAIR experiments. Theoretically, the ability to handle nonzero chemical potential in QCD or at least QCD-like theories is crucial.

We explore, by means of the gauge/gravity duality, the phase structure of strongly coupled non-conformal theories similar to QCD by investigating the physics of probes in the thermal plasmas of these theories with nonzero chemical potential. In a spirit similar to the holographic computation of $\eta / s$ we search for universality in the behavior of heavy quark-antiquark $(Q \bar{Q})$ bound states in large classes of holographic theories. This may yield insight into certain QCD processes relevant for the QGP produced in heavy ion collisions, e.g. suppression of charmonia [8] or bottomonia.

Specifically, we analyze the $Q \bar{Q}$ screening distance, the $Q \bar{Q}$ free energy in the medium (roughly speaking the interaction potential), and the running coupling extracted from the free energy. Previous work on similar problems includes [9] 10] for vanishing chemical potential, and [11,12] for nonzero chemical potential in $\mathscr{N}=4$ supersymmetric Yang-Mills theory. A more detailed account of our findings will be published elsewhere.

We start with the prototype of gauge/gravity duality between classical supergravity on $\mathrm{AdS}_{5}$ and conformal $\mathscr{N}=4$ supersymmetric Yang-Mills theory (SYM) with gauge group $\mathrm{SU}\left(N_{\mathrm{c}}\right)$ in the limit of infinite number of colors, $N_{\mathrm{c}} \rightarrow \infty$, and large ' $\mathrm{t}$ Hooft coupling $\lambda \equiv g_{\mathrm{YM}}^{2} N_{\mathrm{c}}$. A thermal bath for the gauge theory is dual to a black hole in $\mathrm{AdS}_{5}$. Putting charge on the black hole, we can induce a chemical potential in the dual theory. Therefore, as a starting point we consider $\mathrm{AdS}_{5}$ with a ReissnerNordström black hole (AdS-RN), in Poincaré coordinates,

$$
\begin{aligned}
& \mathrm{d} s^{2}=\frac{R^{2}}{z^{2}}\left(-h(z) \mathrm{d} t^{2}+\mathrm{d} \vec{x}^{2}+\frac{\mathrm{d} z^{2}}{h(z)}\right) \\
& \text { with } h(z)=1-\left(1+Q^{2}\right)\left(\frac{z}{z_{\mathrm{h}}}\right)^{4}+Q^{2}\left(\frac{z}{z_{\mathrm{h}}}\right)^{6} \text {. }
\end{aligned}
$$

Here, $R$ is the curvature scale and the black hole horizon is at $z=z_{\mathrm{h}}$. The dual theory is in a thermal state with temperature $T=\left(1-\frac{1}{2} Q^{2}\right) /\left(\pi z_{\mathrm{h}}\right)$ and chemical potential $\mu=\sqrt{3} Q / z_{\mathrm{h}}$. We have $0 \leq Q \leq \sqrt{2}$.

To come closer to real-world physics, we study models in which conformality is explicitly broken by deforming the AdS spacetime. On the one hand, we consider the CGN model proposed by Colangelo, Giannuzzi, and Nicotri [13]. It is specified by the metric (1) with an additional overall warp factor $\mathrm{e}^{c^{2} z^{2}}$ with deformation parameter $c$. On the other hand, we study a family of 1-parameter models which we derive from the action used in [14], which adds to 5-dimensional gravity with metric $g_{\mu \nu}$ and negative cosmological constant a scalar field $\phi$ and a $\mathrm{U}(1)$ gauge field $A_{\mu}$ whose boundary value equals the chemical potential in the dual gauge theory. Our ansatz with deformation parameter $\kappa$ is 


$$
\begin{array}{r}
g_{\mu \nu} \mathrm{d} x^{\mu} \mathrm{d} x^{\nu}=\mathrm{e}^{2 A(z)}\left(-h(z) \mathrm{d} t^{2}+\mathrm{d} \vec{x}^{2}\right)+\frac{\mathrm{e}^{2 B(z)}}{h(z)} \mathrm{d} z^{2}, \\
A(z)=\log \left(\frac{R}{z}\right), \quad \phi(z)=\sqrt{\frac{3}{2}} \kappa z^{2}, \quad A_{\mu} \mathrm{d} x^{\mu}=\Phi(z) \mathrm{d} t,
\end{array}
$$

where $R$ is a constant and $h(z)$ is the redshift factor induced by the black hole. We derive two classes of models from this ansatz, treating $\phi$ as the dilaton or not, called 'string frame' and 'Einstein frame' models, respectively. The solutions for $B, h$, and $\Phi$ can be given in closed form [15]. At fixed $(\mu, T)$, a maximal deformation $\kappa_{\max }$ exists that still allows a black hole solution representing $(\mu, T)$. Since these models solve gravity equations of motion (EOMs) they are expected to be thermodynamically consistent, as opposed to models in which the metric is deformed 'by hand'.

In the CGN model we find unusual behavior in some observables at low temperatures and chemical potentials. Such behavior occurs when we consider moving probes, for example in the case of the drag force. We believe that these artifacts are unphysical. Indeed, they do no longer occur in models obtained as solutions of gravity EOMs. In particular, they are absent in our 1-parameter models. We will report on this in detail elsewhere [16].

\section{Screening Distance}

We study a dipole of an infinitely heavy quark and its antiquark, separated by a distance $L$ in the deconfined plasma of the gauge theory. The quarks are situated at the 4-dimensional boundary ( $z=0$ in our coordinates) and are connected by a macroscopic string in the bulk (see e.g. [4]). We accommodate a finite velocity $v$ of the $Q \bar{Q}$ system with respect to the surrounding medium by boosting the bulk metric with rapidity $\eta=\operatorname{artanh}(v)$. In order to find the classical string configuration we have to extremize the Nambu-Goto action in the given gravity background.

There is a distance $L_{\mathrm{S}}$, such that for $L<L_{\mathrm{S}}$ there are two string configurations connecting the dipole, while no such solution exists for $L>L_{\mathrm{s}}$. Thus, $L_{\mathrm{S}}$ is called the screening distance of the $Q \bar{Q}$ interaction in the thermal medium. In the studies [17, 18] it was found that at any temperature $T$ the screening distance is bounded from below by $L_{\mathrm{S}}^{\mathcal{V}}=4 \mathrm{SYM}(T)$ under consistent deformations of AdS-Schwarzschild, the dual of $\mathscr{N}=4 \mathrm{SYM}$ at $\mu=0$ (where we understand consistency in the sense of solving equations of motion of a suitable 5-dimensional gravity action).

The question arises whether this bound holds under the inclusion of a chemical potential. We find that in the CGN and similar models, depending on (sign) choices in the metric, $L_{\mathrm{S}}$ can change in both directions. More interesting are consistent deformations which in our case are the two classes of 1-parameter models.

As can be seen from Fig. 1 the bound on $L_{\mathrm{S}}$ is violated in the Einstein frame models at large chemical potential, approximately when $\mu \gtrsim \sqrt{\kappa}$. In the string frame models it is only violated for $Q \bar{Q}$ pairs moving sufficiently fast at large $\mu \gtrsim \sqrt{\kappa}$. 
Fig. 1 Rapidity dependence of the screening distance $L_{\mathrm{s}}$ at finite temperature $T$ and chemical potential $\mu$ in the 1-parameter models, evolving from the conformal case (dual to AdS-RN) to the maximal deformation $\kappa_{\max }$. The asymptotic behavior $L_{\mathrm{s}} \propto \cosh ^{-1 / 2}(\eta)$ is scaled out. At large $\eta$, the differences between the two 1-parameter models vanish. $L_{\mathrm{S}}, \mu$, and $\kappa$ are measured in units of temperature.

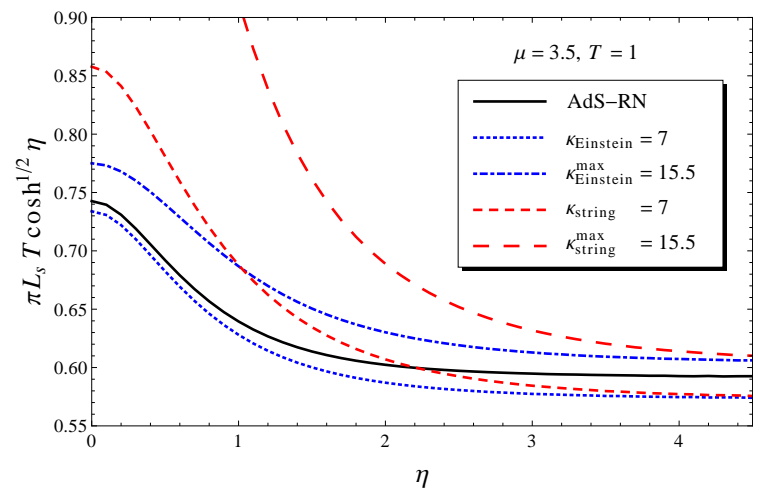

However, for all deformations, the amount of violation of the bound is relatively small such that there might exist a slightly lower, improved bound. Moreover, in the Einstein frame models, the screening distance is a robust observable, depending only weakly on the deformation.

We also study the dependence of $L_{\mathrm{s}}$ on the $Q \bar{Q}$ rapidity $\eta$. Figure 1 illustrates that the ultrarelativistic scaling of the screening distance $L_{\mathrm{S}} \propto \cosh ^{-1 / 2}(\eta)$ is robust and remains valid in all models, at all chemical potentials. It is interesting to note that this robustness against deformation is different from what was found in [11] for other explicitly non-conformal models.

\section{Free Energy and Running Coupling}

The free energy $F(L)$ of the $Q \bar{Q}$ system can be extracted from the extremal classical string action following a well-known procedure (see e.g. [4]). The typical features in the consistently deformed models can be seen in Fig. 2. Here, $F(L)$ is normalized such that a configuration having $F<0$ has less free energy than the non-interacting, unbound $Q \bar{Q}$ system. Thus, we see that the effect of increasing the chemical potential is a decrease in binding energy. Taking this together with our findings concerning the screening distance, we see that in these holographic models an increased net density around the $Q \bar{Q}$ dipole weakens its binding by screening the interaction. We find this effect regardless of the specific model under consideration.

To explore the impact of non-conformality on the interaction in more detail, we study the running coupling defined via the derivative of the free energy,

$$
\alpha_{Q \bar{Q}}(L) \equiv \frac{3}{4} L^{2} \frac{\mathrm{d} F(L)}{\mathrm{d} L}
$$

In the conformal case, where $F(L) \propto 1 / L$ is Coulombic, $\alpha_{Q \bar{Q}}=$ const. Hence, any non-trivial dependence on $L$ measures the deviation from conformality. 
Fig. 2 Typical $Q \bar{Q}$ free energy $F(L)$ in a non-conformal 1-parameter string frame model for varying values of the chemical potential $\mu$. The behavior in the Einstein frame models is qualitatively similar. $\sqrt{\lambda}$ is a constant. The endpoints of the curves are located at the respective screening distances.
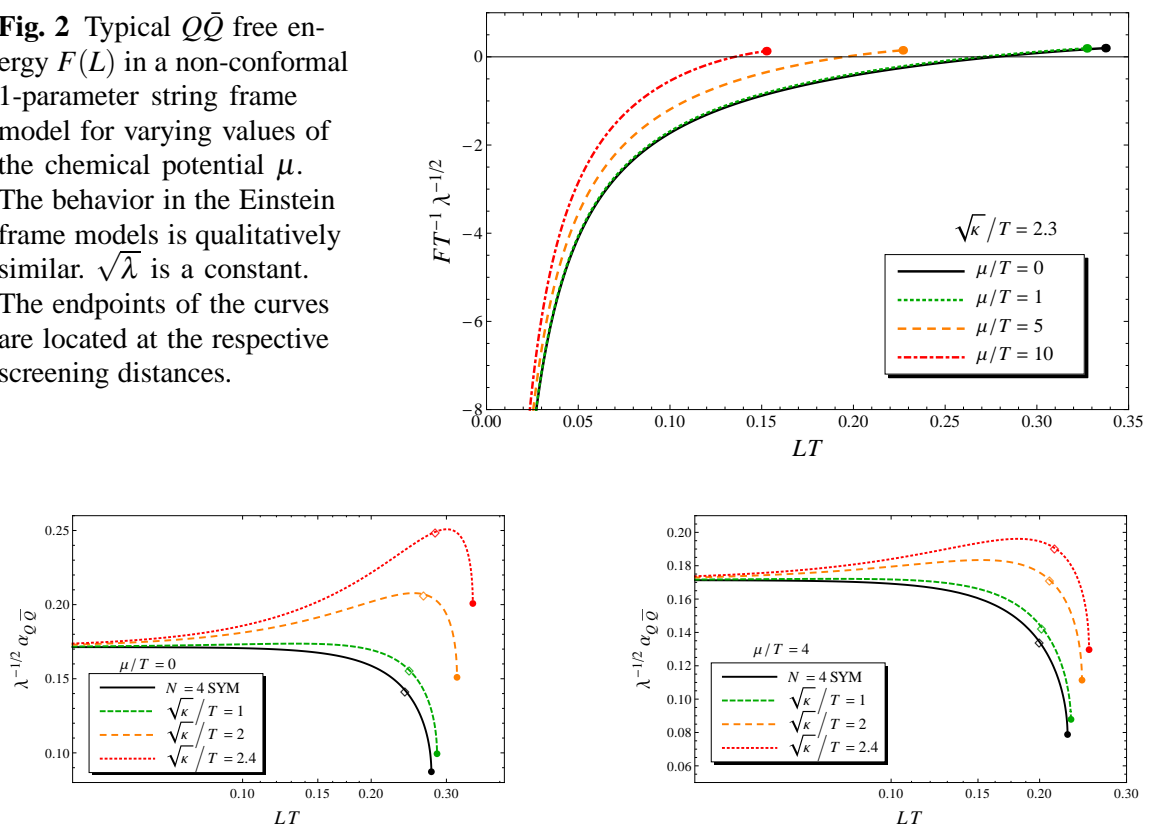

Fig. 3 Running coupling $\alpha_{Q \bar{Q}}(L)$ in $\mathscr{N}=4$ SYM and 1-parameter string frame models with increasing non-conformality parameter $\kappa$, for zero (left) and nonzero (right) chemical potential $\mu$.

In Fig. 3 we show $\alpha_{Q \bar{Q}}(L)$ for the 1-parameter string frame models. The qualitative picture is the same in the Einstein frame models; however, in these models, $\alpha_{Q \bar{Q}}$ is very robust under deformations, so that the curves for different $\kappa$ deviate only very little from each other.

From Fig. 3 we see that $\alpha_{Q \bar{Q}}$ is constant in the UV, i.e. at small distances $L$. One can also see this from the free energy itself in Fig. 2] which approaches Coulombic form in the UV. This is due to the restoration of conformality in the UV, the bulk realization of which is the condition on the metric to be asymptotically AdS.

At larger distance, both the explicit non-conformality and the thermal medium affect the $Q \bar{Q}$ interaction. In effect, the running coupling starts to deviate from its asymptotic value. We find a robust increase above the UV value at intermediate length scales due to non-conformality in all deformed models, at vanishing $\mu$ [17] but also at nonzero chemical potential.

The plasma starts to take effect at the thermal scale, roughly $L_{\mathrm{th}} \sim 1 / T$, leading to a drop-off of $\alpha_{O \bar{O}}$, before the $Q \bar{Q}$ interaction is entirely screened. (The endpoint of the curves $\alpha_{Q \bar{Q}}(\bar{L})$ is the screening distance $L_{\mathrm{s}}$.) This pattern is also found in lattice QCD studies of $\alpha_{Q \bar{Q}}$ in the deconfined phase [19]. While the lattice calculations are presently restricted to vanishing chemical potential due to the sign problem, the holographic models allow us to explore the effect of a chemical potential in strongly coupled QCD-like theories. 
We find that the effect of the chemical potential is relatively weak: the drop-off scale is only weakly dependent on the chemical potential while it strongly depends on the temperature.

\section{Conclusion}

We have reported on some results of our studies of deformed, i.e. non-conformal, gauge/gravity models for strongly coupled plasmas at nonzero chemical potential. Studying large classes of holographic models, we address the problem of the strong coupling dynamics of moving heavy mesons in deconfined plasmas by looking for universality. In particular, we include a nonzero chemical potential $\mu$ in our studies.

We find a certain robustness of the screening distance $L_{\mathrm{s}}$ at nonzero $\mu$ under deformations. However, when switching on the chemical potential, $L_{\mathrm{s}}^{\mathcal{N}}=4 \mathrm{SYM}$ no longer is a lower bound on the screening distance under deformations, unlike in the case of $\mu=0$.

Furthermore, we observe a weak impact of the chemical potential on the qualitative features of the quark-antiquark interaction. Even quantitatively, the dependence of characteristic scales on the chemical potential is generally significantly weaker than their dependence on temperature.

Acknowledgements We thank K. Schade for many helpful discussions. This work was supported by the ExtreMe Matter Institute EMMI.

\section{References}

1. J.M. Maldacena, Adv. Theor. Math. Phys. 2, 231 (1998)

2. S.S. Gubser, I.R. Klebanov, A.M. Polyakov, Phys. Lett. B428, 105 (1998)

3. E. Witten, Adv. Theor. Math. Phys. 2, 253 (1998)

4. J. Casalderrey-Solana, H. Liu, D. Mateos, K. Rajagopal, U.A. Wiedemann, arXiv: 1101.0618 (2011)

5. G. Policastro, D.T. Son, A.O. Starinets, Phys.Rev.Lett. 87, 081601 (2001)

6. P. Kovtun, D.T. Son, A.O. Starinets, JHEP 0310, 064 (2003)

7. P. Kovtun, D.T. Son, A.O. Starinets, Phys. Rev. Lett. 94, 111601 (2005)

8. T. Matsui, H. Satz, Phys. Lett. B178, 416 (1986)

9. H. Liu, K. Rajagopal, U.A. Wiedemann, Phys. Rev. Lett. 98, 182301 (2007)

10. H. Liu, K. Rajagopal, Y. Shi, JHEP 08, 048 (2008)

11. E. Caceres, M. Natsuume, T. Okamura, JHEP 10, 011 (2006)

12. S.D. Avramis, K. Sfetsos, D. Zoakos, Phys. Rev. D75, 025009 (2007)

13. P. Colangelo, F. Giannuzzi, S. Nicotri, Phys. Rev. D83, 035015 (2011)

14. O. DeWolfe, S.S. Gubser, C. Rosen, Phys. Rev. D83, 086005 (2011)

15. A. Samberg, Diploma thesis, Heidelberg University (2012)

16. C. Ewerz, L. Lin, A. Samberg, in preparation

17. K. Schade, PhD thesis, Heidelberg University (2012)

18. C. Ewerz, K. Schade, PoS(Confinement X)270 (2013); C. Ewerz, K. Schade, in preparation

19. O. Kaczmarek, F. Karsch, F. Zantow, P. Petreczky, Phys.Rev. D70, 074505 (2004) 